\title{
Involvement of cytokinins in adventitious and lateral root formation
}

\author{
Takeshi Kuroha* and Shinobu Satoh
}

Graduate School of Life and Environmental Sciences, University of Tsukuba, Tennoudai 1-1-1, Tsukuba, Ibaraki 305-8572, Japan

* Present address: Plant Productivity Systems Research Group, RIKEN Plant Science Center, Tsurumi, Yokohama 230-0045, Japan

Corresponding author: S. Satoh, E-mail: satohshi@sakura.cc.tsukuba.ac.jp, Phone: +81-29-853-4871, Fax: $+81-29-853-4579$

Received on October 17, 2006; Accepted on February 21, 2007

\begin{abstract}
The root systems of higher plant consist of primary, lateral, and adventitious roots. There is a current hypothesis, named 'root apical dominance', that root-derived suppressor negatively regulates the formation of adventitious and lateral roots. According to this hypothesis, we have studied the mechanisms involved in the regulation of adventitious and lateral root formation. trans-zeatin riboside (ZR), one of cytokinin species, was identified in squash root xylem sap as a main suppressor of adventitious root formation in cucumber, suggesting that ZR transported from root via xylem sap acts as an endogenous suppressor of hypocotyl adventitious root formation in planta. Moreover, we isolated Arabidopsis mutant, wooden leg-3 (wol-3), for stimulated adventitious root formation in intact plants. Analyses of wol-3 mutant have revealed that cytokinin receptors are necessary for the primary and lateral root formation via the formation of auxin-transporting vascular tissues in the hypocotyl, but not for adventitious roots. In this review, we discuss the relationship between cytokinin and adventitious and lateral root formation.
\end{abstract}

Keywords: adventitious root, auxin, cytokinin, lateral root, xylem sap

\footnotetext{
Abbreviations: AHK, Arabidopsis histidine kinase; CRE, cytokinin response; DR5, direct repeat 5; EMS, ethyl-methane sulfonate; GUS, $\beta$-glucuronidase; IPT, adenosine phosphate-isopentenyltransferase;

LC/MS/MS, liquid chromatography/tandem mass spectrometry; WOL, wooden leg; Z, trans-zeatin; ZR, trans-zeatin riboside
}

\section{Introduction}

In higher plants, the root system is composed of primary, lateral, and adventitious roots. Adventitious roots are formed from stems or hypocotyls, and lateral roots are formed from primary roots. Adventitious and lateral roots are 'postembryonic' roots, in contrast to the 'embryonic' primary roots. Adventitious and lateral roots cover most of the root system, and can change the architecture of the root system in response to changes in the environment, such as changes in the nutrient or water availability (López-Bucio et al. 2003).

The cutting of hypocotyls and root tissue stimulates the formation of adventitious and lateral roots, respectively. It has been well established that basipetally transported auxin, which accumulates at the basal ends of hypocotyls and stem cuttings, plays a stimulatory role in adventitious root formation (Maldiney et al. 1986, Label et al. 1989, Fig. 1). Similarly, removal of the shoot apex decreases both the level of endogenous auxin in the basal portion of a cutting, and the number of adventitious roots (Nordström and Eliasson 1991). Auxin-overproducing mutants of Arabidopsis produce numerous adventitious and lateral roots from hypocotyls and the primary root of intact plants, respectively (Casimiro et al. 2003). Auxin-insensitive mutants of Arabidopsis are inhibited in lateral root formation (Casimiro et al. 2003). It has been reported that ethylene also stimulates adventitious root formation. Ethylene is synthesized in response to wounding, and promotes adventitious root development in cut tissues. Inhibitors for ethylene biosynthesis and perception reduce the number of adventitious roots (Robbins et al. 1985), and ethylene-insensitive tomato mutants produce fewer adventitious roots in the cutting than

Kuroha T and Satoh S 2007 Involvement of cytokinins in adventitious and lateral root formation. Plant Root 1: 27-33. doi:10.3117/plantroot.1.27

Copyrights 2007, Plant Root (JSRR), www.plantroot.org 


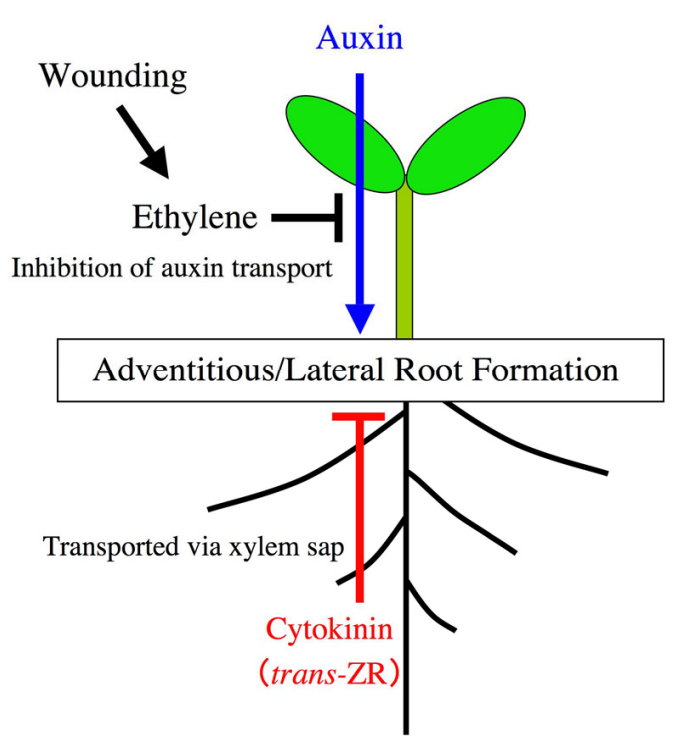

Fig. 1. Schematic representation of the regulation of adventitious and lateral root formation. Lines ending with arrowheads show positive regulation, whereas lines ending in cross-lines indicate negative regulation.

wild-type cuttings (Clark et al. 1999). There is a hypothesis that ethylene interrupts polar auxin transport inside the vascular bundles and the pericycle, and that promoting adventitious and lateral root initiation just above the interruption sites is due to local auxin accumulation (Aloni et al. 2006, Fig. 1). On the other hand, it is also suggested that abscisic acid has distinct roles in lateral root formation, especially in response to water availability (De Smet et al 2006).

There is a current hypothesis that a root-derived suppressor negatively regulates the formation of adventitious and lateral roots (Soekarjo 1965). This hypothesis, termed 'root apical dominance', is analogous to that for apical dominance in shoots. There have been a number of reports on the inhibition of adventitious root formation caused by exogenous treatment with cytokinin (Bollmark and Eliasson 1986). Therefore, it is suggested that cytokinin negatively regulates the formation of lateral and adventitious roots, but the involvement of cytokinin in the root morphogenesis in vivo has not been well understood. This review focused on our recent studies and the related current research progress on the functions of cytokinins in adventitious and lateral root formation.

\section{Inhibition of the adventitious root formation by cytokinin in xylem sap}

It has been expected that a root-derived suppressor(s) is transported to the hypocotyl through the xylem via the transpiration stream. In fact, the fractions in squash root xylem sap inhibited adventitious root formation in the culture of cucumber seedling hypocotyls (Satoh et al. 1998). A significant concentration of a novel amino acid, methoxybenzylglutamine [ $N^{5}$-(4-methoxyphenyl)methyl-L-glutamine], was identified in the inhibitory fraction of the root xylem sap (Satoh et al. 1998). Chemically synthesized methoxybenzylglutamine inhibited the formation of adventitious roots at $5 \times 10^{-3} \mathrm{M}$ but not at $5 \times 10^{-4} \mathrm{M}$ (Satoh et al. 1998). However, the endogenous concentration of methoxybenzylglutamine in root xylem sap has been estimated to be $10^{-6}$ $\mathrm{M}$. This endogenous concentration is far too low to be effective in shoot, suggesting that methoxybenzylglutamine is a poor candidate for the sole suppression of adventitious root formation.

We attempted to identify main endogenous suppressors of the formation of adventitious roots using double-solvent extraction, followed by normal and reverse-phase column chromatography, and analysis by liquid chromatography/tandem mass spectrometry (LC/MS/MS) (Kuroha et al. 2002). The inhibitory activity in squash root xylem sap was observed only in the $20 \%$ acetonitrile eluate of the butanol phase fraction (Satoh et al. 1998). Strong inhibition of the adventitious root formation on hypocotyl was observed in a fraction by reverse and normal phase chromatography. LC/MS/MS analysis showed that a trans-zeatin riboside (ZR), one of cytokinin species, was a major component of this fraction (Kuroha et al. 2002). ZR showed significant inhibitory effects on adventitious root formation at concentrations from 3 x $10^{-9} \mathrm{M}$ to $10^{-4} \mathrm{M}$ (Kuroha et al. 2002). LC/MS/MS analysis showed that the concentration of endogenous $\mathrm{ZR}$ in root xylem sap was estimated to be $2 \times 10^{-8} \mathrm{M}$ (Kuroha et al. 2002). This concentration is sufficient to inhibit the formation of adventitious roots by cucumber hypocotyls. These results suggest that ZR in root xylem sap plays as the main suppressor on the hypothesis of 'root apical dominance', and that ZR transported from roots to shoots via the transpiration stream negatively regulates the formation of lateral and adventitious roots (Fig. 1). This recent hypothesis is named as 'Cytokinin-dependent root apical dominance' (Alnoi et al 2006). Alnoi et al (2005) reported that the expression of the cytokinin response gene and cytokinin staining with monoclonal antibodies decreased in the condition with almost no transpiration, suggesting that cytokinin moves from root to shoot by transpiration stream. ZR was the most abundant cytokinin in root xylem sap in many species such as Urtica dioica (Beck and Wagner 1994) and pea (Beveridge et al. 1997). It is possible that ZR transported from roots to the hypocotyl is converted to trans-zeatin $(\mathrm{Z})$, one of most active cytokinins, and that the $\mathrm{Z}$ performs the inhibition of 
adventitious root formation from hypocotyls. Although the enzyme activity of cytokinin nucleosidase was reported in wheat germ (Chen et al. 1981), the $\mathrm{ZR}$ to $\mathrm{Z}$ conversion mechanism following translocation from roots to shoot is to be elucidated.

It was reported that transgenic tobacco and $\mathrm{Arabi}^{-}$ dopsis plants with reduced cytokinin content, due to the overexpression of a cytokinin oxidase gene, showed an increase in lateral and adventitious roots (Werner et al 2001, Werner et al 2003). Moreover, double knock-out mutants of cytokinin receptors, ARABIDOPSIS HISTIDINE KINASE 2 (AHK2) and $A H K 3$, in Arabidopsis showed the enhanced lateral root formation (Riefler et al 2006). The genes for adenosine phosphate-isopentenyltransferase (IPT) have been isolated, which catalyzes the initial step of cytokinin biosynthesis (Takei et al 2001, Kakimoto 2001). In Arabidopsis, it was reported that AtIPT5 gene expresses in root primordia, columella root caps, and that AtIPT7 gene expresses in endodermis of the root elongation zone (Miyawaki et al 2004). The products of these genes may be involved in the synthesis of ZR in root that may participate in the negative regulation of the adventitious and lateral root formation.

\section{Loss-of-function in cytokinin receptors affects adventitious and lateral root formation}

Although many auxin-related mutants of Arabidopsis showing altered lateral root formation were reported (Casimiro et al. 2003), there had been no reports on the screening of 'root apical dominance' mutants. To explore genetic factors involved in the negative regulation of adventitious root formation, we screened for Arabidopsis mutants with elevated adventitious root formation (Kuroha et al 2006). An M2 population of ethyl-methane sulfonate (EMS)-mutagenized lines of the Arabidopsis was grown and selected the lines displaying an increased number of adventitious roots originating from the hypocotyl. One of them, wol-3, originally $\operatorname{ar}-\mathrm{C} 22$, showed retardation of the primary root growth, no production of lateral roots, and enhanced formation of adventitious roots from hypocotyls (Fig. 2; Kuroha et al. 2006). This means that wol-3 showed reduced root apical dominance in hypocotyls.

To determine the molecular nature of the wol-3 gene product, we identified the gene through positional cloning. The wol-3 locus was mapped to a $250-\mathrm{kb}$ region. Among this region, there is a gene for cytokinin receptor, ARABIDOPSIS HISTIDINE KINASE 4 (AHK4). The AHK4 is also referred to as WOL (Mähönen et al 2000, de León et al 2004) or CYTOKININ RESPONSE 1 (CRE1; Inoue et al 2001), and wol mutants, wol-1 and wol-2, were reported to be retarded growth of the primary root with an aborted vascular system. The nucleotide sequence of the AHK4 locus in the wol-3 mutant was found to contain a mutation. This mutation was a single nucleotide exchange, producing a non-conservative amino acid substitution $\left(\mathrm{M}_{436}\right.$ to $\mathrm{I}_{436}$; Kuroha et al. 2006). To determine whether introduction of the intact AHK4 gene complements the wol-3 mutation, the genome region encompassing the intact $A H K 4$ gene and the putative promoter region was transformed to the wol-3 mutant. The transgenic wol-3 lines showed the wild-type root phenotype, indicating that the AHK4 gene complemented the wol-3 mutant (Kuroha et al. 2006). The wol-3 was identified a new ahk4 allele with a phenotype identical to wol.

It was reported that the AHK4 protein has typical structure of eukaryotic membrane receptor in the two-component regulatory system (Kakimoto 2003). The wol-3 mutation was a single nucleotide exchange in the region between the transmembrane motifs and the histidine kinase domain. The residue in wol-3 mutation is conserved among all cytokinin receptors, suggesting that this residue is important for the function of the AHK4. Three Arabidopsis cytokinin receptors, AHK2, AHK3, and AHK4, have been reported (Kakimoto 2003). Each single mutant in the $A H K 2, A H K 3$ and $A H K 4$ genes except for the wol and cre 1-1 alleles has normal root morphology (Table 1). However, the phenotypes of ahk2 ahk3 ahk4 mutant showing enhanced adventitious roots were quite similar to those of the wol-3 mutant (Kuroha et al 2006). de León et al (2004) reported that wol alleles displayed interallelic complementation, and specu-
A

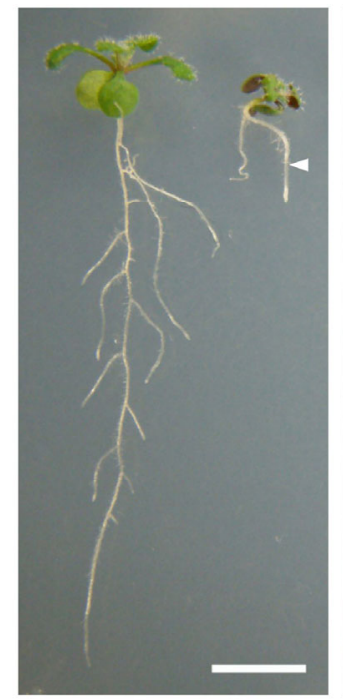

B

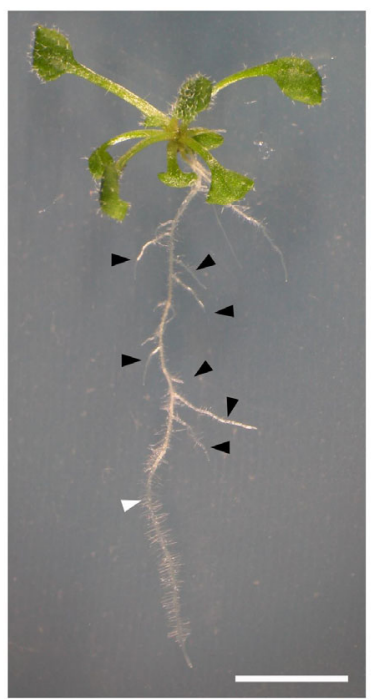

Fig. 2. Root phenotype in the wol-3 mutant. (A) 10-day-old wild type (left) and the wol-3 mutant (right) seedlings. (B) 20-day-old wol-3 mutant seedling. The white arrowheads highlight adventitious roots, and the black arrowheads point to secondary adventitious roots. Scale bars: $5 \mathrm{~mm}$. 
lated that the wol mutation leads to the inhibition of dimerization. In cytokinin receptors, it is suggested that only AHK4 has strong phosphatase activity on the phosphorelay network (Mähönen et al 2006). Moreover, it is reported that the $A H K 4$ is expressed more strongly than $A H K 2$ or $A H K 3$ in the root procambium, which forms primary xylem in primary vascular tissues (Mähönen et al. 2000, Mähönen et al 2006). The different expression levels and the functions among the cytokinin receptors might lead the different root phenotype of the mutants for the cytokinin receptors.

\section{Relationship between development of auxin transporting vascular tissues and adventitious and lateral root formation}

The wol allele of the ahk4 mutant has a reduced number of vascular initials that subsequently form xylem, but not phloem cells, suggesting that AHK4 appears to play an important role in vascular morphogenesis (Mähönen et al. 2000). However, this cannot explain why the mutant developed a normal vascular system in adventitious root. We observed the details of the hypocotyl phenotype of the wol-3 mutant with a light microscopy. Five days after germination, the amount of vascular tissue in the middle and lower parts of the hypocotyl was significantly reduced, while it was normal in the upper part of the hypocotyl (Kuroha et al 2006). The vascular system of the wol-3 hypocotyl contains only xylem cells, similar to that of the wol-3 primary root (Kuroha et al 2006).

It was reported that basipetally transported auxin from shoot stimulates adventitious and lateral root formation (Nordström and Eliasson 1991, Casimiro et al 2001). Therefore, we speculated that the adventitious root-forming wol-3 phenotype was caused by extensive auxin accumulation in the hypocotyl. To investigate the relationship between the auxin response and the adventitious-root-forming wol-3 phenotype, we introduced the DR5 (DIRECT REPEAT 5)::GUS fusion gene into wild-type Columbia and the wol-3 mutant, and compared the GUS expressions. The DR5 is the synthetic auxin responsive promoters known as useful tools for monitoring auxin responses in planta. Although wild-type Arabidopsis hypocotyls showed slight GUS expression, the wol-3 hypocotyls showed strong expression around the aborted vascular tissue (Kuroha et al 2006). Strong GUS expression in hypocotyl vascular tissue was observed when the wild-type hypocotyl was cut surgically (Kuroha et al 2006). These results suggest that in the wol-3 mutant, the decreased cell numbers in the xylem parenchyma of the hypocotyl leads to the accumulation of auxin and the activation of adventitious root formation from the hypocotyl (Fig. 3).

Table 1. AHK4 mutants and their root phenotypes in the normal condition

\begin{tabular}{|c|c|c|c|c|c|c|}
\hline \multirow{2}{*}{ Allele name } & \multirow{2}{*}{ Mutagen } & \multicolumn{3}{|c|}{ Mutation site } & \multirow{2}{*}{ Phenotype } & \multirow{2}{*}{ Reference } \\
\hline & & Nucleotide & Amino acid & Domain & & \\
\hline wol-1 & EMS & the 3 rd exon & $\mathrm{T} 278 \mathrm{I} \dagger$ & Extracellular & + & Mähönen et al (2000) \\
\hline wol-2 & EMS & the 6th exon & L506F & Histidine kinase & + & de León et al (2004) \\
\hline wol-3 & EMS & the 5 th exon & M436I & Other: & + & Kuroha et al (2006) \\
\hline ahk4-1 & T-DNA & the 8th intron & & & - & Ueguchi et al (2001) \\
\hline crel-1 & EMS & the 5 th exon & G467D & Histidine kinase & + & Inoue et al (2001) \\
\hline crel-2 & T-DNA & the 1st exon & & & - & Inoue et al (2001) \\
\hline crel-3 & EMS & the 9 th exon & W1003stop & 2 nd receiver & - & Franco-Zorrilla et al (2002) \\
\hline crel-4 & EMS & the 9th exon & T985I & 2 nd receiver & - & Franco-Zorrilla et al (2002) \\
\hline crel-5 & EMS & & & Other§ & - & Franco-Zorrilla et al (2002) \\
\hline crel-6 & EMS & $\begin{array}{l}\text { the last nucleotide } \\
\text { just before the } 6 \text { th } \\
\text { intron }\end{array}$ & G470R & Histidine kinase & - & Franco-Zorrilla et al (2002) \\
\hline crel-7, 8 & EMS & the 5 th exon & Q452stop & Histidine kinase & - & Franco-Zorrilla et al (2002) \\
\hline crel-9 & EMS & the 9th exon & A1009T & 2 nd receiver & - & Franco-Zorrilla et al (2002) \\
\hline cre $1-10$ & T-DNA & the 8th intron & & & - & Higuchi et al (2004) \\
\hline cre1-11 & T-DNA & the 5 th exon & & & - & Higuchi et al (2004) \\
\hline crel-12 & T-DNA & the 2 nd intron & & & - & Higuchi et al (2004) \\
\hline
\end{tabular}

+ , retarded growth of the primary root with an aborted vascular system; -, normal growth of the primary root.

$\dagger$ T278I means isoleusine (I) substitution of threonine (T) 278.

the region between the $\mathrm{C}$ terminal transmembrane motif and the histidine kinase domain.

$\S$ near $\mathrm{N}$ terminal transmembrane motif. Blank in Mutation site indicates no description in the reference. 


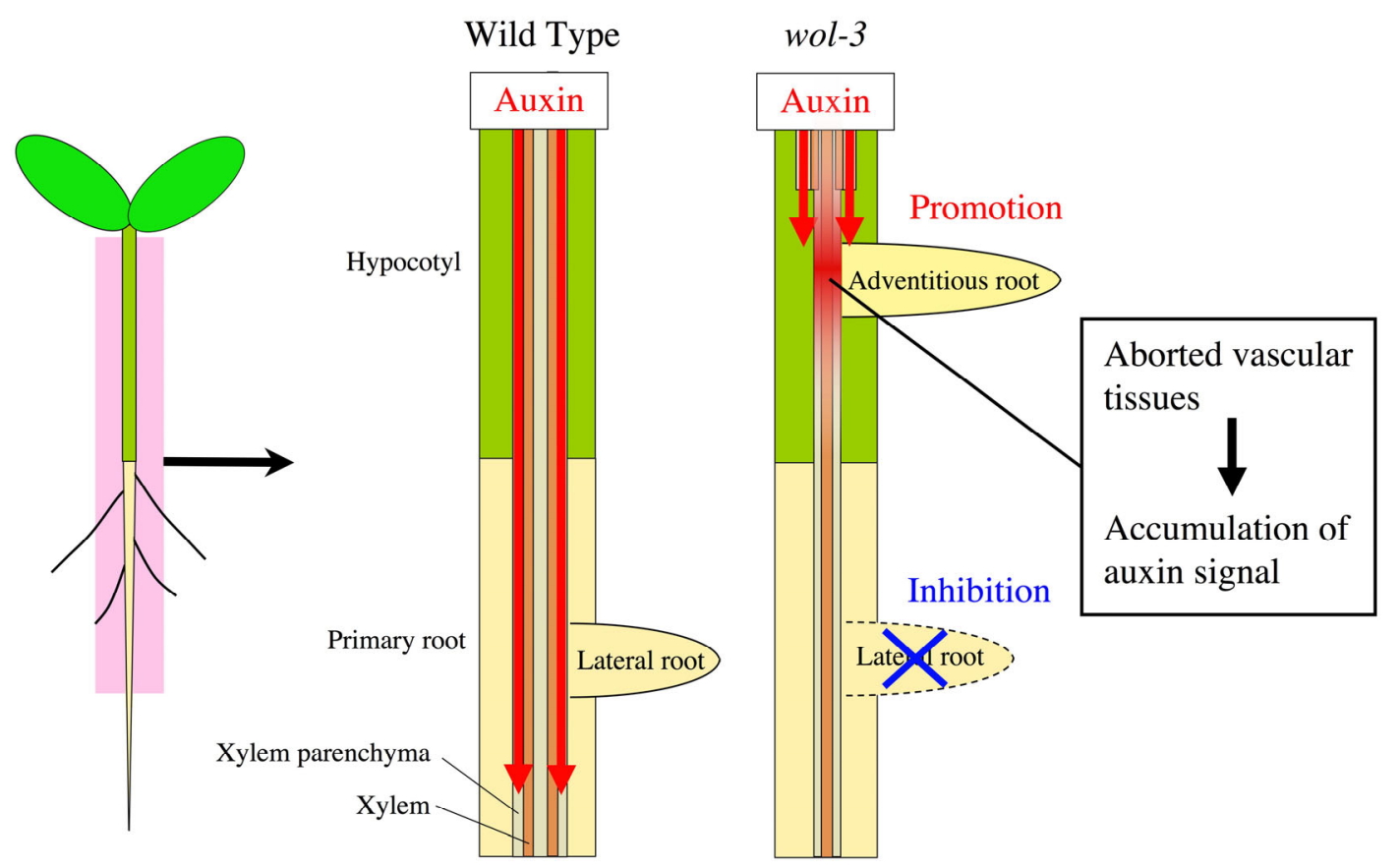

Fig. 3. Schematic representation of root and hypocotyl phenotypes in the wol-3 mutant. Red arrows represent auxin transport from hypocotyls to roots. In the wild type seedling, auxin normally transports from hypocotyls to root. In the wol-3 hypocotyl, the decreased cell numbers in the xylem and xylem parenchyma of the hypocotyls leads to the inhibition of auxin transport, the accumulation of auxin signal, the adventitious root formation from the hypocotyls. The inhibition of auxin transport in the wol-3 hypocotyl leads to the inhibition of lateral root formation.

The application of auxin to the wol-3 primary root resulted in the induction of DR5::GUS expression and subsequent lateral root formation (Kuroha et al 2006), suggesting that the auxin response in the wol-3 primary root is normal. When the wol-3 primary root was cut off, both the expression of DR5::GUS and lateral root formation were inhibited (Kuroha et al 2006). Furthermore, basipetal auxin transport from the hypocotyl to the primary root in the wol-3 mutant was significantly inhibited compared to the wild type (Kuroha et al 2006). These results suggest that the inhibition of lateral root formation in the wol-3 mutant is not caused by the reduction of cell numbers in the vascular systems of the primary root, but results from the inhibition of auxin transport from the hypocotyls (Fig. 3). Interestingly, the wol-3 and ahk2 ahk3 ahk4 mutants developed a normal vascular system in adventitious roots, although the hypocotyl and primary root developed aborted vascular tissues (Kuroha et al 2006). This suggests that the cytokinin receptors AHK2, 3, 4 are not involved in the development of vascular tissue in the adventitious roots. In addition, it is also suggested that the development of leaves, which supply more auxin than only the cotyledon, enables the wol-3 mutant to develop the normal vascular tissue and branch their laterals in the adventitious roots.

\section{Concluding remarks}

In our study, we showed that ZR, one species of cytokinin, in xylem sap plays as the main suppressor of the formation of adventitious roots. We also reported that cytokinin receptors are necessary for the formation of auxin-transporting vascular tissues in hypocotyls, and that it is necessary for lateral root, but not for adventitious root, suggesting that there are different mechanisms for the development of vascular tissues between in primary and lateral roots and in adventitious roots. Genetic studies on the system of the cytokinin synthesis, signaling, and translocation will help us to understand the detailed mechanism of the lateral and adventitious root formation. It is expected that cytokinin become effective tool for modifying the architecture of the root system in crops.

\section{Acknowledgments}

We thank Dr. Shigeo Yoshida, Dr. Tadao Asami, Dr. Hisashi Kato, Chiharu Ueguchi, and Dr. Hitoshi Sakakibara for the invaluable collaboration work. This work was supported, in part, by Grants-in-Aid for Scientific Research on Priority Area (no. 14036204) and the "Research for the Future" 
Program, of the Japanese Society for the Promotion of Science (JSP-RFTF97L00601).

\section{References}

Aloni R, Aloni E, Langhans M, Ullrich CI 2006 Role of cytokinin and auxin in shaping root architecture: regulating vascular differentiation, lateral root initiation, root apical dominance and root gravitropism. Ann. Bot. 97: 883-93.

Aloni R, Langhans M, Aloni E, Dreieicher E, Ullrich CI 2005 Root-synthesized cytokinin in Arabidopsis is distributed in the shoot by the transpiration stream. J. Exp. Bot. 56: 1535-44.

Beck E, Wagner B 1994 Quantification of the daily cytokinin transport from the root to the shoot of Urtica dioica L. Bot. Acta 107: 342-348.

Beveridge C, Murfet I, Kerhoas L, Sotta B, Miginiac E, Rameau C 1997 The shoot controls zeatin riboside export from pea roots. Evidence from the branching mutant rms4. Plant J. 11: 339-345.

Bollmark M, Eliasson L 1986 Effects of exogenous cytokinins on root formation in pea cuttings. Physiol. Plant. 68:662-666.

Casimiro I, Beeckman T, Graham N, Bhalerao R, Zhang H, Casero P, Sandberg G, Bennett MJ 2003 Dissecting Arabidopsis lateral root development. Trends Plant Sci. 8: 165-171.

Casimiro I, Marchant A, Bhalerao RP, Beeckman T, Dhooge S, Swarup R, Graham N, Inzé D, Sandberg G, Casero PJ, Bennett M 2001 Auxin transport promotes Arabidopsis lateral root initiation. Plant Cell 13:843-852.

Chen C-M, Kristopeit SM 1981 Metabolism of cytokinin: Deribosylation of cytokinin ribonucleoside by adenosine nucleosidase from wheat germ. Plant Physiol. 68:1020-1023.

Clark DG, Gubrium EK, Barrett JE, Nell TA, Klee HJ 1999 Root formation in ethylene-insensitive plants. Plant Physiol. 121: 53-60.

de León BG, Zorrilla JM, Rubio V, Dahiya P, Paz-Ares J, Leyva A 2004 Interallelic complementation at the Arabidopsis CRE1 locus uncovers independent pathways for the proliferation of vascular initials and canonical cytokinin signalling. Plant J. 38: 70-79.

De Smet I, Zhang H, Inzé D, Beeckman T 2006 A novel role for abscisic acid emerges from underground. Trends Plant Sci. 11:434-439.

Franco-Zorrilla JM, Martin AC, Solano R, Rubio V, Leyva A, Paz-Ares J 2002 Mutations at CRE1 impair cytokinin-induced repression of phosphate starvation responses in Arabidopsis. Plant J. 32: 353-360.

Higuchi M, Pischke MS, Mähönen AP, Miyawaki K, Hashimoto Y, Seki M, Kobayashi M, Shinozaki K, Kato T, Tabata S, Helariutta Y, Sussman MR, Kakimoto T 2004 In planta functions of the Arabidopsis cytokinin receptor family. Proc. Natl. Acad. Sci. USA. 101:8821-8826.

Inoue T, Higuchi M, Hashimoto Y, Seki M, Kobayashi M, Kato T, Tabata S, Shinozaki K, and Kakimoto T 2001 Identification of CRE1 as a cytokinin receptor from Arabidopsis. Nature 409: 1060-1063.

Kakimoto T 2001 Identification of plant cytokinin biosynthetic enzymes as dimethylallyl diphosphate:ATP/ADP isopentenyltransferases. Plant Cell Physiol 42: 677-685.

Kakimoto T 2003 Perception and signal transduction of cytokinins. Annu. Rev. Plant Biol. 54:605-27.
Kuroha T, Kato H, Asami T, Yoshida S, Kamada H, Satoh S 2002 A trans-zeatin riboside in root xylem sap negatively regulates adventitious root formation on cucumber hypocotyls. J. Exp. Bot. 53: 2193-2200.

Kuroha T, Ueguchi C, Sakakibara H, Satoh S 2006 Cytokinin receptors are required for normal development of auxin-transporting vascular tissues in the hypocotyl but not in adventitious roots. Plant Cell Physiol. 47: 234-243.

Label P, Sotta B, Miginiac E 1989 Endogenous levels of abscisic acid and indole-3-acetic acid during in vitro rooting of wild cherry explants produced by micropropagation. Plant Growth Regul. 8: 325-333.

López-Bucio J, Cruz-Ramirez A, Herrera-Estrella L 2003 The role of nutrient availability in regulating root architecture. Curr. Opin. Plant Biol. 6: 280-287.

Miyawaki K, Matsumoto-Kitano M, and Kakimoto T 2004 Expression of cytokinin biosynthetic isopentenyltransferase genes in Arabidopsis: tissue specificity and regulation by auxin, cytokinin, and nitrate. Plant J. 37:128-138.

Mähönen AP, Bonke M, Kauppinen L, Riikonen M, Benfey PN, and Helariutta Y 2000 A novel two-component hybrid molecule regulates vascular morphogenesis of the Arabidopsis root. Genes Dev. 14: 2938-2943.

Mähönen AP, Higuchi M, Tormakangas K, Miyawaki K, Pischke MS, Sussman MR, Helariutta Y, Kakimoto T 2006 Cytokinins regulate a bidirectional phosphorelay network in Arabidopsis. Curr. Biol. 16:1116-22.

Maldiney R, Pelèse F, Pilate G, Sotta B, Sossountzov L, Miginiac E 1986 Endogenous levels of abscisic acid, indole-3-acetic acid, zeatin and zeatin-riboside during the course of adventitious root formation in cuttings of Craigella and Craigella lateral suppressor tomatoes. Physiol. Plant. 68: 426-430.

Nordström A-C, Eliasson L 1991 Levels of endogenous indole-3-acetic acid and indole-3-acetylaspartic acid during adventitious root formation in pea cuttings. Physiol Plant. 82: 599-605.

Riefler M, Novak O, Strnad M, Schmülling T 2006 Arabidopsis cytokinin receptor mutants reveal functions in shoot growth, leaf senescence, seed size, germination, root development, and cytokinin metabolism. Plant Cell 18: 40-54.

Robbins J, Reid M, Paul J, Rost T 1985 The effect of ethylene on adventitious root formation in mung bean (Vigna radiata) cuttings. J. Plant Growth Regul. 4: 147-157

Satoh S, Kuroha T, Wakahoi T, Inouye Y 1998 Inhibition of the formation of adventitious roots on cucumber hypocotyls by the fractions and methoxybenzylglutamine from xylem sap of squash foot. J. Plant Res. 111: 541-546.

Soekarjo R 1965 On the formation of adventitious roots in cuttings of Coleus in relation to the effect of indoleacetic acid on the epinastic curvature of isolated petioles. Acta Bot. Neerl. 14: 373-399.

Takei K, Sakakibara H, Sugiyama T 2001 Identification of genes encoding adenylate isopentenyltransferase, a cytokinin biosynthesis enzyme, in Arabidopsis thaliana. J. Biol. Chem. 276: 26405-26410.

Ueguchi C, Sato S, Kato T, Tabata S 2001 The AHK4 gene involved in the cytokinin-signaling pathway as a direct receptor molecule in Arabidopsis thaliana. Plant Cell Physiol. 42: 751-755.

Werner T, Motyka V, Laucou V, Smets R, Van Onckelen H, Schmülling T 2003 Cytokinin-deficient transgenic Arabidopsis plants show multiple developmental alterations indicating opposite functions of cytokinins in 
the regulation of shoot and root meristem activity. Plant Cell 15: 2532-2550.

Werner T, Motyka V, Strnad M, Schmülling T 2001 Regulation of plant growth by cytokinin. Proc. Natl. Acad. Sci. U. S. A. $98: 10487-10492$.

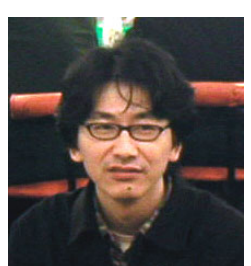

Dr. Takeshi Kuroha's research interests are lateral and adventitious root formation, cytokinin transport and biosynthesis, and long-distance signaling between aboveorgan and root in plant. 